\title{
Hybrid solar-gas-electric dryer optimization with genetic algorithms
}

\author{
El Ferouali, H. ${ }^{\text {; }}$ Gharafi, M. ${ }^{\text {b }}$ Zoukit, A. ${ }^{\text {; }}$ Doubabi, S. ${ }^{\text {a }}$ Abdenouri, N. ${ }^{\text {** }}$ \\ ${ }^{a}$ LSET, Cadi Ayyad University, Marrakech, Morocco. \\ b Department of Mathematics, Laboratory of Applied Mathematics and Computer Science, Cadi \\ Ayyad University, Marrakech, Morocco. \\ *E-mail of the corresponding author: n.abdenouri@uca.ma
}

\begin{abstract}
To promote the hybrid solar dryers for use even under unfavorable weather and to overcome the intermittance state issue, the energy consumption should be optimized and the response time should be reduced. This work concerns a drying chamber connected to a solar absorber where the air can be heated also by combustion of gas and by electric resistance. To optimize the control parameters, an evolutionary optimization algorithm simulating natural selection was used. It was combined with a predictive model based on the artificial neural networks (ANN) technique and used as a fitness function for the genetic algorithm (GA). The ANN is a learning algorithm that needs training through a large dataset, which was collected using CFD simulation and experimental data. Then a GA was executed in order to optimize two objectives: The energy consumption and the t95\% response time in which the drying chamber temperature reaches its set point $\left(60^{\circ} \mathrm{C}\right)$. After optimization, a 30\% decrease of the $195 \%$ response time, and $20 \%$ decrease of the energy consumption were obtained.
\end{abstract}

Keywords: hybrid solar dryer; artificial neural network; temperature regulation; energy consumption; genetic algorithm. 


\section{Introduction}

The solar drying process offers a promising alternative way of preservation foods and crops at a favorable energy cost unlike conventional energy sources. However, at night and in the cloudy time, the dehydration process slows extending the drying time. Even more, multiple rehydration of products harms their quality. Hybrid version of solar dryers can offer a wide respond to a wider demand. It reduces energy costs and the drying process is ensured in spite of the alternation of the climatic conditions. The hybrid dryer can ensure a continuous and controlled drying process by using a secondary energy sources [2].

The electric heating resistance was combined to solar air heater in previous works where a prototype was designed in a favorable conditions for optimized power consumption [3]. Unfortunately, this dryer is efficient just for the low air flow rates. In fact, the energy contribution by using the auxiliary heater increased from $74.92 \%$ to $86.78 \%$ by increasing the air flow rate from $1 \mathrm{~m} / \mathrm{s}$ to $2 \mathrm{~m} / \mathrm{s}$.

The auxiliary heating system based on gas combustion has been studied in many cases. It was employed in a tunnel dryer by using a heat exchanger connected the gas burners and placed inside the drying chamber [4]. In addition, López-Vidaña et al. [5] suggested putting the burner inside the drying chamber. However, in most cases, the flue gases released by combustion affect the dried products.

In this work, a heat exchanger placed inside the solar collector with a separated exhaust of flue gases was considered. This new configuration seems to be practical, safe and easy to handle.

Modeling of the dryer is one of the most important aspects of drying technology. Most of these models were established by using several techniques such as: computational fluid dynamics (CFD), artificial neural network, Fuzzy, thermal modeling and energy balance mathematical modeling [6]. Computational Fluid Dynamics CFD is usually used to model and predict the temperature and velocity fields in the studied area.

The aim of this work is to optimize the control parameters in order to reduce the cost and the response time of drying. This case requires multi-objective optimization of a relatively complex system. Genetic algorithms are best suited for such application, since they allow the approximation of global optimums while avoiding local ones[7]. The flip side of a genetic algorithm is that it need a large number of design points for an accurate estimate output parameters. The design points were noted by using CFD tools as a predictive method of the dryer [8]. 


\section{Materials and Methods}

\subsection{The hybrid solar-gas-electric dryer}

The hybrid solar-gas dryer consists of a solar-gas collector where the air is heated by solar radiation and by 9 gas burners fitted in the collector (Fig.1). Geometric and thermophysical specifications of the solar-gas collector are given in [9]. The electric heating consists of two electric resistances of 2000W each one, positioned inside the drying chamber (Fig. 2). The drying chamber's volume is $1 \mathrm{~m}^{3}$, and its walls are insulated by $5 \mathrm{~cm}$ of mineral fiber.



Fig. 1 Solar-gas collector.

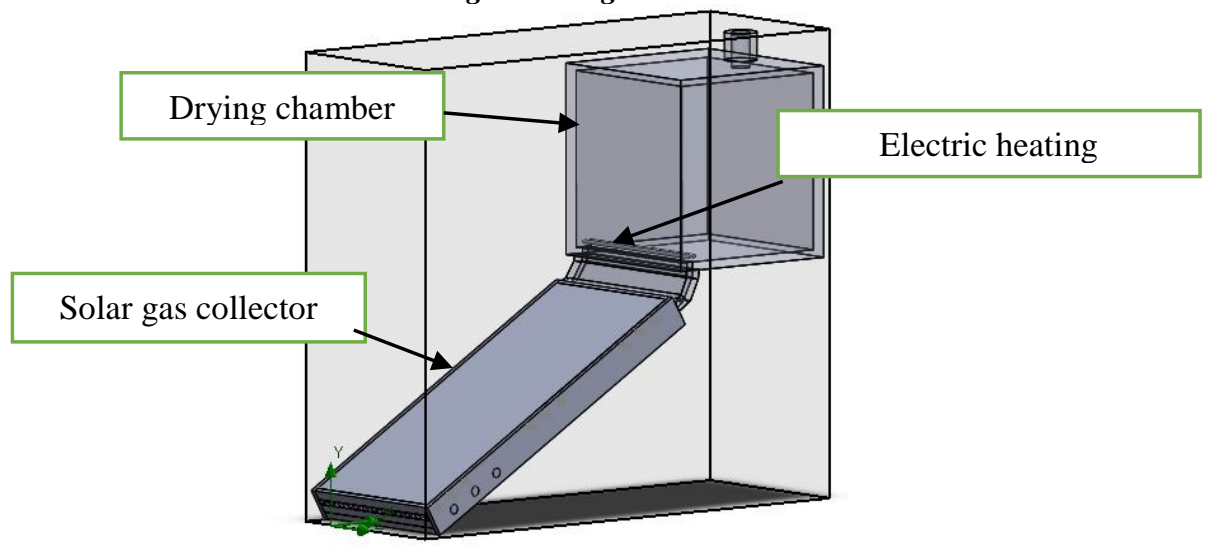

Fig. 2 Design and simulation of the hybrid solar-gas-electric dryer.

\subsection{CFD modeling}

3D simulations were done on SolidWorks Flow Simulation. The software solves NavierStokes equations with the Finite Volume Method (FVM). The $\kappa-\varepsilon$ model was used in this calculation. Solar radiation is modeled to be perpendicular to the glass of the solar-gas collector. Whereas, the flames of the burners are modeled as volume heat sources located under a metallic sheet (exchanger). The electric auxiliary heater is modeled by two volume 
heat sources of $2 \mathrm{KW}$ each as maximum value. Simulations were performed in forced convection at an air flow rate of $0.025 \mathrm{~kg} / \mathrm{s}$ in transient state for $90 \mathrm{~min}$. The input parameters are solar radiation $\left[0-900 \mathrm{~W} / \mathrm{m}^{2}\right]$, gas power $[0-5000 \mathrm{~W}]$, and electric power [0$4000 \mathrm{~W}]$. The mean drying chamber temperature was controlled at $60^{\circ} \mathrm{C}$. Hence, the electric and gas heaters are not always activated during the time dependent simulation. Their activation is dependent on the mean drying chamber air temperature that represents the goal value. They were turned on until the goal value is bit greater than the specified control value $\left(60^{\circ} \mathrm{C}\right)$. The value of the dead band is considered as equal to $3^{\circ} \mathrm{C}$, Thus, the volume heat sources is turned off until the control value became lower than $57^{\circ} \mathrm{C}$.

The following specifications were used in the CFD modeling:

The solar-gas collector is oriented southward under an angle of $30^{\circ}$ versus the horizontal.

- The model uses the discrete ordinates (DO) radiation model.

- The wind velocity is equal to $5 \mathrm{~m} / \mathrm{s}$ and its direction is $40^{\circ}$. Ambient temperature is equal to $25^{\circ} \mathrm{C}$.

- Local refinement of the mesh is used in this model in order to take into account the peculiarities of the model's geometry; the mesh is composed from 304179 cells.

\subsection{Predictive modeling and optimization}

A genetic algorithm (GA) was used to optimize the control parameters. This method starts by creating a population of random members of the same species as the optimized object. Then, the performance of each member of the population was evaluated according to fitness function. Upon the evaluation phase members with higher scores proceed to the crossover operation, where their control parameters can be transferred to next generation. To monitor the (GA) optimization process, the overall score of the population were tracked, and its distribution along with the best fit score. This case corresponds to a multiobjective optimization: quality $(q)$, response time $(t)$ and the cost $(c)$. Many variants of the (GA) were used in order to get the best results, the Table 1 shows the used options in each variant:

Table 1. Genetic algorithms configurations.

\begin{tabular}{ccccc}
\hline (GA) ID & Fitness function & Population size & Mutation rate & Best fit criterion \\
\hline 1 & Min $(\mathrm{q}, \mathrm{t}, \mathrm{c})^{*}$ & 2000 & 0.3 & Lowest score \\
2 & ${\text { RMS }(\mathrm{Q}, \mathrm{T}, \mathrm{C})^{*}}_{3}$ & 2000 & 0.4 & Lowest score \\
3 & Centroid of 3 objectives* & 500 & 0.2 & Lowest score \\
4 & Sum of the objectives & 500 & 0.2 & Lowest score \\
& scores & & & \\
\hline
\end{tabular}

*q, t, c: output of the dryer's performance, the normalized to the worst possible output.

${ }^{*} \mathrm{Q}, \mathrm{T}, \mathrm{C}$ : the raw output of the dryer's performance.

*The centroid method is based on a geometric tool which is the CENTROID of equilateral triangle, where its summits are the objectives. Each summit is weighted by the performance of the dryer in the objective. The fitness function is evaluated by calculating the distance of 
a performance output centroid and the geometrical center. Loweer the distance is, better the members fits the solution. The fit is evaluated as follows:

Let $q, t$ and $c$ be the quality ratio, the time response ratio and the cost ratio respectively:

$$
\begin{gathered}
q=1-\frac{\text { Absolute error }}{\text { Maximum Absolute error }} \\
t=1-\frac{\text { Response time }}{\text { Drying time }} \\
c=1-\frac{\text { Energy invested }}{\text { Energy of full power functionning }}
\end{gathered}
$$

The Euclidian distance of the generated point to the geometrical center of the triangle can be written as:

$$
d=\frac{1}{q+t+c} \sqrt{\frac{1}{4}(c-t)^{2}+3\left(\frac{q}{3}-\frac{t+c}{6}\right)^{2}}
$$

The implementation of the genetic algorithm was done on MatLab, using the "GA" built in function. However, this method requires the evaluation of the output of a large number of control points. Since a CFD simulation response time is inadmissible big, the Artificial Neural Networks (ANN) method developed here is more suitable to predict the solar dryer response for each input parameters. A neuron is fundamentally a node that accepts weights inputs and fires an output from 0 to 1 depending on whether the weighted sum of the inputs exceeds a threshold or not. The threshold is called the bias of the neuron. The neural networks method is a learning algorithm, it consists of changing the weights of each neuron proportionally to their contribution to the error and with smoothing ratio, called learning rate. It can be used for both regression and classification, but according to our modeling problem, it performs well in classification. To do so, an encoding method was established to transform a floating value into one hot binary string. Which is a string of zeros and one in one spot, this spot indicate the class of the value.

For instance, assuming that a temperature variable is within a range of 10 to $50^{\circ} \mathrm{C}$, the value of $25^{\circ} \mathrm{C}$ can be represented as: 0100 , with a bin of $10^{\circ} \mathrm{C}$. To define a deep neural net, the following arguments are required: The number of layers, the number of nodes per layer, the learning rate, the feed forward batch size, the number of epochs, the cost function and the optimizer.

In our case, a neural network of two terminal layers (input and output) was used. In addition to that we defined 3 hidden layers. The numbers of nodes per layer are respectively 
3, 350, 400, 350, 30. The dataset size is 27000 record. $10 \%$ of this data is used for testing. The number of runned epochs in a training session is 100000 epoch. AdamOptimizer was used to change the weights of the nodes with a learning rate of 0.001 . In order to avoid the overfitting problem, the data was shuffled every epoch. The implementation of the neural network model was done with tensorflow running on a CPU. The algorithm was written in Python. The average training time was about 72 hours on a machine of the following specifications: 8Gb of memory, Intel i7 Quad Core processor of a processing frequency of $3.40 \mathrm{GHz}$.

\section{Results and discussion}

The proposed method consists of generating data using CFD simulation along with experimental results to train a neural network model. The generated data was validated against the experimental data, the reached accuracy level was about 95\%. The size of the dataset collected is 27000, with 7 columns: solar radiation, electric power, gas power, absolute error on the temperature inside the dryer, the t95\% response time, and the electric and gas energy invested.

The generated data is then transformed in order to train a classification model based on the neural networks method, the method allowed us to predict the performance output data in any control point in a few hundred milliseconds with an accuracy of 95\%. The generated data is then transformed in order to train a classification model based on the neural networks method, the method allowed us to predict the performance output data in any control point in a few hundred milliseconds with an accuracy of 95\%. Fig 3 shows the evolution of the accuracy during the training session. The accuracy in the beginning of the training is very low, but as the steps increase the accuracy increases but at a decelerating rate.

The predictive model was exported and served to the genetic algorithm written in MatLab to execute the optimization code, the Table 2 shows the results for 4 cases using the sum of the objectives as fitness function which yielded the best results.

The average gain on the cases studied is about 20\%. it is calculated assuming that the electric energy cost approximately twice as the gas energy. The following formula is used to evaluate the gain on the cost after optimization:

$$
G_{\text {cost }}=0.6 \times \frac{E_{\text {opt } E l e c}}{E_{\text {Elec }}}+0.3 \times \frac{E_{\text {opt gas }}}{E_{\text {gas }}}
$$



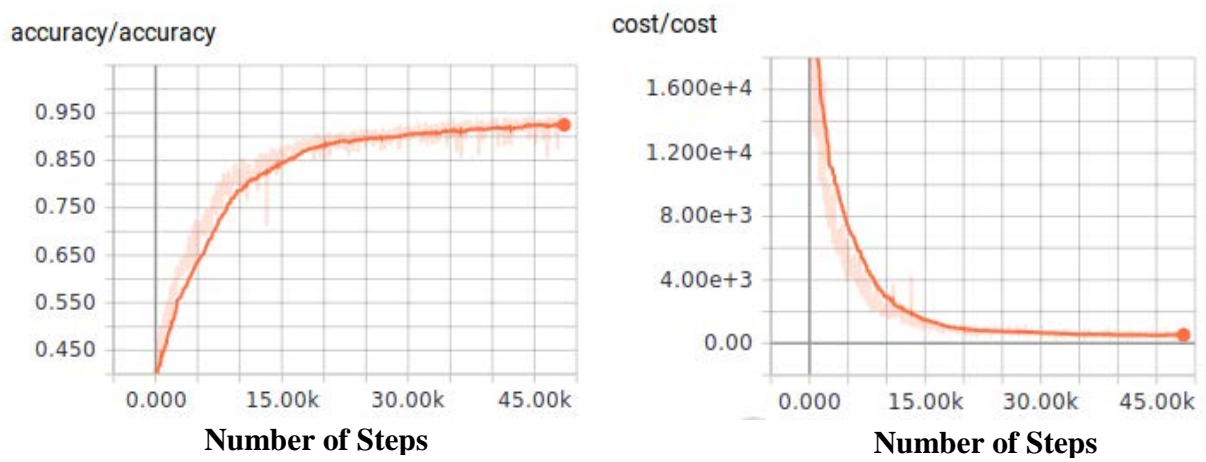

Fig. 3 cost and accuracy of the predictive model during a training session.

Table 2. results of the optimization

\begin{tabular}{lllllll}
\hline $\begin{array}{l}\text { Solar } \\
\text { radiation }\end{array}$ & $\begin{array}{l}\text { Electric } \\
\text { Power } \\
(\mathbf{W})\end{array}$ & $\begin{array}{l}\text { Gas } \\
\text { power } \\
(\mathbf{W})\end{array}$ & $\begin{array}{l}\text { Absolute } \\
\text { Error } \\
\left({ }^{\circ} \mathbf{C}\right)\end{array}$ & $\begin{array}{l}\text { Response } \\
\text { Time } \\
(\mathbf{m i n})\end{array}$ & $\begin{array}{l}\text { Electric } \\
\text { energy } \\
(\mathbf{W h})\end{array}$ & $\begin{array}{l}\text { Gas } \\
\text { Energy } \\
(\mathbf{W h})\end{array}$ \\
\hline 900 & 2543.0 & 5000.0 & 0.5 & 2.0 & 607.5 & 1803.75 \\
500 & 3982.0 & 5000.0 & 0.5 & 2.3 & 948.0 & 1470.0 \\
200 & 4000.0 & 4152.0 & 2.0 & 4.0 & 3199.0 & 2963.0 \\
0 & 2411.0 & 5000.0 & 1.2 & 6.0 & 2015.0 & 3946.0 \\
\hline
\end{tabular}

\section{Conclusions}

In this paper an optimization method of a solar dryer performance is proposed. A predictive model was built with artificial neural networks technique. In order to generate the training dataset for the learning session of the model, several CFD simulations were conducted to determine the gas and electric consumptions, and the $95 \%$ response time of the dryer while its drying chamber temperature is controlled at $60^{\circ} \mathrm{C}$. After the training session, the predictive model reached an admissible level of accuracy about 95\%, in about 50000 epochs. Then a genetic algorithm was performed in order to optimize two main goals that were defined as follow: The energy consumption and the t95\% response time in which the drying chamber temperature reaches its set point $\left(60^{\circ} \mathrm{C}\right)$. The size of the population is 500 , with a mutation chance of $2 \%$. ANN model was used to calculate the fitness function. After 150 generations, we obtained a $30 \%$ decrease of the t95\% response time, and $20 \%$ decrease of the energy consumption.

\section{Nomenclature}

$\begin{array}{ll}q & \text { Quality ratio } \\ t & \text { Reponse time ratio } \\ c & \text { Energy cost ratio } \\ G_{\text {cost }} & \text { Gain on the energy cost after optimization }\end{array}$




$\begin{array}{lll}E_{\text {Opt,Elec }} & \text { Consumed electric energy after optimization } & \text { (Wh) } \\ E_{\text {Elec }} & \text { Consumed electric energy before optimization } & \text { (Wh) } \\ E_{\text {Opt,Gas }} & \text { Consumed gas energy after optimization } & \text { (Wh) } \\ E_{\text {Gas }} & \text { Consumed gas energy before optimization } & \text { (Wh) }\end{array}$

\section{Acknowledgements}

This work was supported by the research institute IRESEN and all of the authors are grateful to the IRESEN for its cooperation.

\section{References}

[1] Basunia, M.A.; Abe, T. Thin-layer solar drying characteristics of rough rice under natural convection. Journal of food engineering 2001, 47(4), 295-301.

[2] Reyes, A.; Mahn, A.; Vásquez, F. Mushrooms dehydration in a hybrid-solar dryer, using a phase change material. Energy Conversion and Management 2014, 83, 241248.

[3] Boughali, S.; Benmoussa, H.; Bouchekima, B.; Mennouche, D. ; Bouguettaia, H.; \& Bechki, D. Crop drying by indirect active hybrid solar-Electrical dryer in the eastern Algerian Septentrional Sahara. Solar Energy 2009, 83(12), 2223-2232.

[4] Oueslati, H.; Mabrouk, S. B.; Marni, A. Design and installation of a solar-gas tunnel dryer: Comparative experimental study of two scenarios of drying. In Proceedings of the 5th International Renewable Energy Congress (IREC) 2014, 1-6.

[5] López-Vidaña, E. C.; Méndez-Lagunas, L. L.; Rodríguez-Ramírez, J. Efficiency of a hybrid solar-gas dryer. Solar Energy 2013, 93, 23-31.

[6] Prakash, O.; Laguri, V.; Pandey, A.; Kumar, A.; Kumar, A. Review on various modelling techniques for the solar dryers. Renewable and Sustainable Energy Reviews 2016, 62, 396-417.

[7] Ahmadi, M.H.; Ahmadi, M.A.; Feidt, M. Performance optimization of a solar-driven multi-step irreversible brayton cycle based on a multi-objective genetic algorithm. Oil \& Gas Science and Technology-Revue d’IFP Energies nouvelles 2016, 71 (1), 16.

[8] Wang, T.;Gao, H.; Qiu, J. A combined adaptive neural network and nonlinear model predictive control for multirate networked industrial process control. IEEE Transactions on Neural Networks and Learning Systems 2016, 27 (2), 416-425.

[9] El Ferouali, H.; Zoukit, A.; Salhi, I.; Doubabi, S.; Abdenouri, N.; \& El Kilali, T. Heat transfer in a solar-gas-electric hybrid dryer for the control of its operation and for energy management and storage. In Electrical Sciences and Technologies in Maghreb (CISTEM) IEEE, 1-6. 